\title{
Aclimatização de mudas de bananeira em substratos contendo resíduo de mineração de areia
}

\author{
Edson Shigueaki Nomura ${ }^{\text {; }}$ Eduardo Jun Fuzitani ${ }^{2}$; Valéria Augusta Garcia ${ }^{1}$; \\ Erval Rafael Damatto Junior ${ }^{3}$; Gleidson Alvaro Mariotto ${ }^{4}$
}

\section{RESUMO}

Para o rápido crescimento de mudas de bananeiras produzidas por micropropagação é necessário a utilização de substratos com adequadas características físicas, químicas e biológicas. Objetivou-se avaliar os atributos químicos e físicos de diferentes substratos contendo resíduo de mineração de areia e seu efeito sobre o desenvolvimento de mudas micropropagadas de bananeira 'Grande Naine', dispostos em delineamento em blocos ao acaso, com sete substratos e quatro repetições. Os materiais utilizados foram: resíduo de mineração de areia (RMA), Rendimax Floreira ${ }^{\circledR}$ $\left(\mathrm{RF}^{\circledR}\right)$ e casca de arroz carbonizada (CAC), com os quais se formulou os substratos: S1-100\%RMA; S2-50\% RMA:50\% $\mathrm{RF}^{\circledR}$; S3-50\% RMA:50\% CAC; S4-50\% CAC:50\% RF ${ }^{\circledR}$; S5-25\% RMA:37,5\% CAC:37,5\% RF ${ }^{\circledR}$ S6-50\% RMA:25\% CAC: $25 \% \mathrm{RF}^{\circledR} ; \mathrm{S} 7-75 \% \mathrm{RMA}: 12,5 \% \mathrm{CAC}: 12,5 \% \mathrm{RF}^{\circledR}$. Amostras dos materiais e dos substratos foram separadas para análises químicas e físicas. Noventa e oito dias após o transplante avaliou-se a altura, o diâmetro do colo, o número de folhas e a massa da matéria seca da parte área e das raízes das plantas. Os pHs dos substratos se encontraram na faixa adequada para o cultivo de plantas em recipientes. A CE diminuiu à medida que se aumentou a dose de RMA nos substratos. Os substratos $\mathrm{S} 2, \mathrm{~S} 6$ e o $\mathrm{RF}^{\circledR}$ apresentaram resultados superiores para a densidade seca. Os substratos $\mathrm{S} 2$, S4, S5, S6 e S7 forneceram plantas superiores em altura e diâmetro do colo e, acrescidos do substrato S1, forneceram plantas superiores também no número de folhas e massa de matéria seca da parte aérea. Conclui-se que o RMA pode ser recomendado para a aclimatização de mudas micropropagadas de bananeira 'Grande Naine', desde que usado em mistura com outros materiais ou substratos, com limite máximo de $75 \%$ de inclusão.

Palavras-chave: Musa spp., matéria orgânica, propagação.

\section{ABSTRACT}

\section{Mining sand residue in banana plantlet acclimatization}

Banana is the most cultivated fruit in tropical countries and one of the most consumed in the world. To reach fast development, quality and sanity of banana plants, it is necessary the use of adequate substrate with physical, chemical and biological characteristics for the acclimatization of banana plantlets produced by micropropagation. This study evaluated the effect of different substrates on the development of banana 'Grand Naine' platlets. The experiment was arranged in a randomized block design with seven treatments and four replications. The substrates tested were: T1: $100 \%$ of sand residue, T2: $50 \%$ of sand residue $+50 \%$ of Rendimax Floreira ${ }^{\circledR}$, T3: $50 \%$ of sand residue $+50 \%$ of carbonized rice hull, T4: $50 \%$ of carbonized rice hull $+50 \%$ of Rendimax Floreira ${ }^{\circledR}$, T5: $25 \%$ of sand residue $+37.5 \%$ of

\footnotetext{
Recebido para publicação em 08/10/2010 e aprovado em 08/06/2011

${ }^{1}$ Engenheiros-Agrônomos, Mestres, Agência Paulista de Tecnologia dos Agronegócios - APTA Pólo Vale do Ribeira, Rodovia BR 116, Km 460, Caixa Postal 122, 11900-000, Registro/SP, Brasil.edsonnomura@apta.sp.gov.br; val.garcia@uol.com.br

${ }^{2}$ Engenheiro-Agrônomo, Mestrando, Agência Paulista de Tecnologia dos Agronegócios - APTA Pólo Vale do Ribeira, Rodovia BR 116, Km 460, Caixa Postal 122, 11900-000, Registro/SP, Brasil. edufuzitani@apta.sp.gov.br

${ }_{3}^{3}$ Engenheiro-Agrônomo, Doutor, Agência Paulista de Tecnologia dos Agronegócios - APTA Pólo Vale do Ribeira, Rodovia BR 116, Km 460, Caixa Postal 122, 11900-000, Registro, SP, Brasil. erval@apta.sp.gov.br

${ }^{4}$ Graduando do curso de Agronomia da Universidade Estadual Paulista - Campus Experimental de Registro. Rua Nelson Brihi Badur, 430 - Vila Tupy, 11.900-000, Registro, SP, Brasil.gleimariotto@yahoo.com.br
} 
carbonized rice hull $+37.5 \%$ Rendimax Floreira ${ }^{\circledR}, \mathrm{T} 6: 50 \%$ of sand residue $+25 \%$ of carbonized rice hull $+25 \%$ of Rendimax Floreira ${ }^{\circledR}, \mathrm{T} 7: 75 \%$ of sand residue $+12.5 \%$ of carbonized rice hull $+12.5 \%$ Rendimax Floreira ${ }^{\circledR}$. The variables evaluated included: plant height, pseudostem diameter, leaf number and dry mass of plant roots. All growth data were significant, indicating that mixtures with a maximum proportion of $75 \%$ of mining sand residue can be recommended for the acclimatization of banana 'Grand Naine' plantlets.

Key words: Musa spp., organic matter, propagation.

\section{INTRODUÇÃO}

O Brasil destaca-se entre os principais produtores de bananas, ocupando, em 2008, a quarta colocação mundial, atrás somente da Índia, China e Filipinas (FAOSTAT, 2010). Segundo o IBGE (2010), a produção nacional de bananas alcançou 7,1 milhões de toneladas, cultivados em 514 mil hectares, no ano de 2008. No Estado de São Paulo, a principal região produtora concentra-se no Vale do Ribeira, com produção em 3.483 propriedades, ocupando área de cultivo de 36,2 mil hectares (LUPA, 2008). Nesta região paulista, o cultivo de bananeiras é realizado em propriedades de pequeno porte, o que torna a cultura importante para fixação do homem no campo e na geração de emprego e renda.

Tradicionalmente, a bananeira é propagada por rizomas, porém, os novos plantios comerciais têm empregado mudas micropropagadas em laboratório, utilizando meio asséptico, com a vantagem delas serem isentas de pragas e doenças, além do seu maior vigor e facilidade no transporte e plantio (Lemos et al., 2001). Essa técnica de propagação proporciona alta eficiência, quando comparada com outros métodos de multiplicação da bananeira, já que apresenta rendimento de 150 a 300 mudas por matriz, no período de seis a oito meses (Borges et al., 1997).

As mudas oriundas da micropropagação são comercializadas pelos laboratórios com cerca de dez centímetros de altura e, se plantadas diretamente no campo, podem apresentar elevado índice de perdas, uma vez que essas plantas permaneceram em cultivo totalmente controlado, necessitando-se adaptá-las gradativamente às condições ambientais naturais, bem como ao clima adverso, ao ataque de pragas e doenças, à competição com plantas daninhas e, até mesmo, ao aterramento na ocorrência de fortes chuvas (Sousa et al., 2000). Para essa adaptação, é necessário que as plantas sejam submetidas a um período de aclimatização, podendo este ser sob viveiro sombreado (redução de luminosidade de $50 \%$ ), em recipientes com substratos que propiciem rápido crescimento inicial das mudas, antes do transplantio para o local definitivo. O cultivo em recipientes durante a aclimatização requer irrigações e fertilizações frequentes e, para tanto, faz-se necessário o conhecimento das propriedades químicas e físicas dos substratos, por serem fatores determinantes no manejo e controle de qualidade dos cultivos (Schmitz et al., 2002).

Em vista do aumento da utilização de mudas micropropagadas no cultivo de bananeiras, faz-se necessária a utilização de substratos que permitam satisfatória sobrevivência e desenvolvimento dessas mudas durante a aclimatização. Os substratos podem ser de origem mineral, orgânica ou sintética, misturados ou não, mas devem apresentar características físicas, químicas e biológicas adequadas, que possibilitem rápido crescimento da muda e proporcionem adequada produção de massa de matéria seca nas partes aérea e radicular (Yamanishi et al., 2004).

No Rio Grande do Sul, solo natural ou mistura de solo com areia são utilizados por viveiristas de mudas frutíferas, floríferas e plantas ornamentais, em razão da alta disponibilidade e baixo custo (Gauland, 1997). Porém, há a necessidade de acrescentar outros materiais para propiciar melhores condições de desenvolvimento das mudas.

No mercado, podem ser encontrados diversos substratos comerciais, empregados para a produção de mudas de boa qualidade, porém seu custo é elevado. Neste sentido, uma alternativa poderia ser o aproveitamento de resíduos agroindustriais regionais, encontrados em abundância, de fácil obtenção e de baixo custo de aquisição (Kämpf, 2000a). Atualmente, os produtores de mudas de bananeira da região do Vale do Ribeira SP vêm utilizando, para a sua aclimatização, substrato comercial acrescido de casca de arroz carbonizada e terra de subsolo, sendo que este último, apesar de ter grande disponibilidade e baixo custo, consiste em material heterogêneo e seu acentuado uso pode provocar grandes perdas de solo de áreas agrícolas. 
Diante do exposto, o objetivo deste trabalho foi avaliar os atributos químicos e físicos de diferentes substratos contendo resíduo de mineração de areia e seu efeito sobre o desenvolvimento de mudas micropropagadas de bananeira 'Grande Naine'.

\section{MATERIAL E MÉTODOS}

O experimento foi conduzido no sítio Oriente, localizado às margens da Rodovia Regis Bittencourt, município de Pariquera-Açu, SP. O clima, segundo a classificação de Köppen, é Af: tropical chuvoso, sem estação seca, com a precipitação média do mês mais seco superior a $60 \mathrm{~mm}$. Dados de uma série de oito anos (2000 - 2008), registrados na Estação Experimental de Pariquera-Açu, mostram que a temperatura média anual neste local foi de $21,9^{\circ} \mathrm{C}$ e a pluviosidade média foi de $1.649,7 \mathrm{~mm}$ anuais.

Foi utilizado delineamento em blocos ao acaso, com sete substratos, quatro repetições e cinco plantas por parcela, sendo que as mudas de bananeira 'Grande Naine' foram provenientes de micropropagação, produzidas no laboratório do Pólo Regional Vale do Ribeira, da Agência Paulista de Tecnologia dos Agronegócios (APTA).

Os materiais utilizados para a composição dos substratos foram: resíduo de indústria de mineração de areia, substrato comercial Rendimax Floreira ${ }^{\circledR}$ e casca de arroz carbonizada (CAC). Nas indústrias de mineração de areia, é gerado um resíduo do processo de dragagem do leito do rio, no qual água e areia são conduzidas para as margens, separadas em diferentes granulometrias e depositadas em montes para a retirada do excesso de água. Este excedente de água, juntamente com os materiais em suspensão, é levado por canaletas até atingir uma lagoa ou tanque de decantação, onde os materiais sólidos ficam depositados e a água retorna ao rio, com qualidade. Esse processo não apresenta riscos de contaminação do lençol freático por componentes contaminantes, uma vez que o material decantado é composto exclusivamente por argila, silte e areia. Geralmente, este resíduo acumulado não tem destino, apresentando potencial na composição de substratos para a produção de mudas. O Rendimax Floreira ${ }^{\circledR}$ consiste em substrato comercial composto principalmente por casca de Pinus decomposta. E a CAC é obtida por meio de sua queima sem a presença de chama, produzindo um material leve e poroso, quando utilizado em mistura no substrato, permite boa aeração e drenagem e, livre de plantas daninhas, nematóides e patógenos, não necessita de tratamento químico para esterilização, em razão de ter sido esterilizada no processo de carbonização. Este material pode ser usado, puro ou em mistura com outros substratos, para formação de mudas de diversas espécies de plantas florestais, frutíferas, hortícolas e ornamentais.

A partir destes materiais foram formulados os seguintes substratos: $\mathrm{S} 1-100 \%$ resíduo; S2: $50 \%$ resíduo $+50 \%$
Rendimax Floreira $^{\circledR} ; \mathrm{S} 3: 50 \%$ resíduo $+50 \%$ CAC; S4: 50\% $\mathrm{CAC}+50 \%$ Rendimax Floreira $^{\circledR} ; \mathrm{S} 5: 25 \%$ resíduo $+37,5 \%$ $\mathrm{CAC}+37,5 \%$ Rendimax Floreira ${ }^{\circledR} ; \mathrm{S} 6: 50 \%$ resíduo $+25 \%$ CAC $+25 \%$ Rendimax Floreira ${ }^{\circledR} ;$ e S7: $75 \%$ resíduo $+12,5 \%$ CAC $+12,5 \%$ Rendimax Floreira ${ }^{\circledR}$. Antes do preparo dos substratos, foram retiradas amostras dos materiais para as análises químicas e físicas (Tabela 1 e 2). No preparo dos substratos, a mistura dos materiais nos diferentes tratamentos foi devidamente homogeneizada e, posteriormente, retiraram-se, também, amostras para análises químicas e físicas.

Explantes retirados de meristemas apicais de rebentos de bananeira, devidamente desinfetados, foram acondicionados para adaptação em meio nutritivo MS (Murashige \& Skoog, 1962) com benzilaminopurina (BAP) e ácido 2,4 diclorofenoxiacético (2,4D), previamente autoclavado. Após 20 dias no escuro e 20 dias sob iluminação artificial e temperatura controlada, os explantes foram transferidos para o meio nutritivo MS com BAP, para a multiplicação das plântulas, nas quais ocorreram brotações laterais (3 a 6). A cada 30 dias, realizou-se repicagem ou individualização dessas plântulas por, no máximo, cinco vezes. Posteriormente, as plântulas foram transferidas para enraizamento em frascos com meio MS. Após o enraizamento, as plântulas foram retiradas dos frascos e suas raízes foram lavadas para a retirada do excesso de meio de cultura aderido. As plântulas foram transplantadas em canaletas de $40 \mathrm{~cm}$ de largura e $20 \mathrm{~cm}$ de profundidade, preenchidas com substrato composto por vermiculita e Sunshine, para aclimatização em ambiente protegido, coberto com filme de polietileno transparente. Sessenta dias após o transplante, quando as mudas apresentavam cerca de dez centímetros de altura e cinco folhas, foram arrancadas e plantadas em sacos de polietileno preto $(15 \times 15 \mathrm{~cm})$, preenchidos com cerca de 1,5 L dos diferentes substratos, e mantidos sob tela de sombreamento de $50 \%$. Os sacos foram dispostos sobre a superfície, coberta com filme de polietileno preto, para evitar que as raízes atingissem o solo, mantendo-se espaçamento de $20 \mathrm{~cm}$ entre os sacos. Trinta e cinco dias após o plantio realizou-se adubação de cobertura com a aplicação de 7,5 $\mathrm{g} \mathrm{L}^{-1}$ de substrato do adubo NPK, da fórmula 10-10-10.

As irrigações foram realizadas três vezes por semana, de acordo com as necessidades das plantas e condições climáticas, por meio de microaspersores. O controle fitossanitário foi realizado conforme as recomendações para a cultura (Moreira, 1999), de maneira uniforme em todas as parcelas.

Noventa e oito dias após o plantio, foram avaliadas as seguintes variáveis: altura da planta $(\mathrm{cm})$ com a utilização de régua graduada, tomando-se como referência a distância do colo da planta até a inserção da última folha completamente desenrolada; diâmetro do colo $(\mathrm{cm})$, com a uti- 
lização de paquímetro; número de folhas; massa da matéria seca da parte aérea e das raízes das plantas (g).

Os dados foram submetidos à análise de variância e, quando se observaram diferenças significativas, as médias foram comparadas pelo teste de Tukey a 5\% de probabilidade, utilizando-se o programa estatístico SISVAR (Ferreira, 2003).

\section{RESULTADOS E DISCUSSÃO}

Os resultados das análises químicas e físicas dos diferentes materiais e substratos são apresentados nas Tabelas 1 e 2, em que se observa que os materiais utilizados na composição dos substratos tiveram influência sobre os resultados nos diferentes substratos, de acordo com as diferentes proporções dos materiais.

Observa-se, na Tabela 1, que os valores de $\mathrm{pH}$ dos substratos variaram de 5,3 a 6,2, ficando dentro da faixa ideal recomendada por Kämpf (2000b), que indicou valores ideais entre 6,0 e 7,0 para substratos minerais. Isto propiciou adequada disponibilidade de nutrientes, não ocasionando problemas nutricionais para as mudas de bananeira.

Os valores da condutividade elétrica apresentaram tendência de diminuição, à medida que se aumentou a dose de resíduo na mistura: $\mathrm{S} 5$ (25\% resíduo) $=1,30 \mathrm{dS} \mathrm{m}^{-1} ; \mathrm{S} 6(50 \%$ resíduo $=0,70 \mathrm{dS} \mathrm{m}^{-1} ;$ e S7 (75\% resíduo) $=0,50 \mathrm{dS} \mathrm{m}^{-1}$, em razão da baixa concentração de sais neste material e também na CAC $\left(0,10 \mathrm{dS} \mathrm{m}{ }^{-1}\right)$ (Tabela 1$)$. De acordo com os padrões de condutividade elétrica para o cultivo de plantas ornamentais, determinados por Ballester-Olmos (1993), que considera o valor de $0,75 \mathrm{dS} \mathrm{m}^{-1}$ como muito baixo; 0,75 a 2,00 dS m ${ }^{-1}$ como ideal para sementeiras e mudas em bandejas; 2,00 a 3,50 dS m $\mathrm{m}^{-1}$ apropriado para a maioria das plantas e acima de $3,50 \mathrm{dS} \mathrm{m}^{-1}$ muito alto, os substratos $\mathrm{S} 2$, S4 e S5 encontravam-se na faixa ideal de condutividade elétrica para sementeiras e mudas em bandejas. Isto se deve ao fato de que nestes tratamentos acrescentaram-se maiores quantidades de Rendimax Floreira ${ }^{\circledR}$, contribuindo para a manutenção da condutividade elétrica, pois,

Tabela 1. Análises químicas dos materiais e substratos utilizados para aclimatização de mudas de bananeira 'Grande Naine'

\begin{tabular}{|c|c|c|c|c|c|c|c|c|c|c|c|c|c|}
\hline $\begin{array}{l}\text { Materiais/ } \\
\text { Substratos }^{1}\end{array}$ & pH & $\begin{array}{c}\mathbf{C E}^{2} \\
\mathrm{dS} \mathrm{m}^{-1}\end{array}$ & $\mathrm{NO}_{3}^{-}$ & $\mathrm{NH}_{4}^{+}$ & $\mathbf{P}$ & $\mathbf{K}$ & $\mathbf{C a}$ & $\begin{array}{r}\mathbf{M g} \\
-\mathrm{mg} \mathrm{L}^{-1}\end{array}$ & $\mathbf{S}$ & B & $\mathbf{F e}$ & Mn & Zn \\
\hline Floreira $^{\circledR}$ & 5,4 & 1,9 & 91,7 & 0,3 & 0,26 & 66,3 & 87,4 & 124,0 & 155,4 & 0,21 & 0,02 & 0,02 & 0,02 \\
\hline CAC & 7,6 & 0,1 & $<0,01$ & 1,0 & 16,78 & 86,4 & 2,0 & 1,7 & 13,2 & 0,02 & 0,31 & 0,06 & 0,02 \\
\hline $\mathrm{S} 1$ & 5,3 & 0,1 & $<0,01$ & 3,6 & 0,05 & 2,6 & 41,5 & 16,3 & 64,6 & 0,09 & 3,49 & 0,84 & 0,03 \\
\hline $\mathrm{S} 2$ & 6,0 & 0,9 & 42,9 & 5,6 & 0,18 & 33,1 & 45,6 & 53,7 & 71,4 & 0,08 & 0,02 & 0,05 & 0,02 \\
\hline S3 & 6,2 & 0,4 & 0,6 & 0,8 & 0,02 & 45,7 & 18,5 & 9,2 & 38,0 & 0,02 & 0,13 & 0,01 & 0,01 \\
\hline S4 & 5,9 & 1,2 & 37,0 & 3,8 & 0,47 & 105,3 & 39,9 & 61,1 & 102,9 & 0,10 & 0,03 & 0,01 & 0,01 \\
\hline S5 & 5,9 & 1,3 & 42,9 & 4,2 & 0,15 & 89,1 & 51,6 & 68,0 & 108,7 & 0,13 & 0,03 & 0,05 & 0,01 \\
\hline S6 & 5,9 & 0,7 & 26,2 & 3,1 & 0,01 & 41,2 & 33,6 & 34,9 & 59,7 & 0,06 & 0,05 & 0,05 & 0,02 \\
\hline S7 & 5,9 & 0,5 & 8,3 & 1,0 & 0,01 & 17,2 & 35,2 & 18,7 & 41,2 & 0,04 & 0,04 & 0,03 & 0,02 \\
\hline
\end{tabular}

${ }^{1}$ S1: $100 \%$ resíduo; S2: 50\% resíduo + 50\% Rendimax Floreira ${ }^{\circledR}$; S3: 50\% resíduo + 50\% CAC; S4: 50\% CAC + 50\% Rendimax Floreira ${ }^{\oplus}$; S5: 25\% resíduo + 37,5\% CAC + 37,5\% Rendimax Floreira ${ }^{\circledR} ;$ S6: 50\% resíduo + 25\% CAC + 25\% Rendimax Floreira ${ }^{\circledR} ;$ S7: 75\% resíduo + 12,5\% CAC + 12,5\% Rendimax Floreira ${ }^{\circledR}$. Método de extração: 1:1,5 (Holanda). Métodos de determinação: N-(amoniacal e nitrato): destilação; $\mathrm{K}, \mathrm{Ca}, \mathrm{Mg}, \mathrm{P}, \mathrm{S}, \mathrm{Fe}, \mathrm{Mn}, \mathrm{Zn}$ : ICP-OES; C orgânico: Walkley-Black; ${ }^{2} \mathrm{CE}$ : Condutividade elétrica. Fonte: Centro de Solos e Recursos Agroambientais - Laboratório de Análise de Solo e Planta, IAC - Campinas, SP, 2007.

Tabela 2. Análises físicas dos materiais e substratos utilizados para aclimatização de mudas de bananeira 'Grande Naine'

\begin{tabular}{|c|c|c|c|c|}
\hline \multirow{2}{*}{ Materiais/ Substratos ${ }^{1}$} & CRA $^{2}$ & $\mathbf{D U}^{3}$ & $\mathbf{D S}^{4}$ & \multirow{2}{*}{$\begin{array}{c}\text { Umidade } \\
\%\end{array}$} \\
\hline & $\%(\mathrm{~m} / \mathrm{m})$ & \multicolumn{2}{|c|}{$\mathrm{kg} \mathrm{m}^{-3}-$} & \\
\hline Floreira $^{\circledR}$ & 120,3 & 686,0 & 299,8 & 43,7 \\
\hline CAC & 136,2 & 258,6 & 107,8 & 41,7 \\
\hline S1 & 58,0 & $1.089,1$ & 169,9 & 15,6 \\
\hline $\mathrm{S} 2$ & 69,0 & $1.021,2$ & 338,0 & 33,1 \\
\hline S3 & 86,9 & 870,6 & 247,3 & 28,4 \\
\hline S4 & 107,2 & 525,5 & 228,6 & 43,5 \\
\hline S5 & 91,1 & 711,7 & 228,5 & 32,1 \\
\hline S6 & 86,7 & 902,3 & 287,8 & 31,9 \\
\hline S7 & 70,1 & $1.034,4$ & 247,2 & 23,9 \\
\hline
\end{tabular}

${ }^{1}$ S1: $100 \%$ resíduo; S2: 50\% resíduo + 50\% Rendimax Floreira ${ }^{\circledR}$; S3: 50\% resíduo + 50\% CAC; S4: 50\% CAC + 50\% Rendimax Floreira ${ }^{\oplus}$; S5: $25 \%$ resíduo $+37,5 \%$ CAC $+37,5 \%$ Rendimax Floreira ${ }^{\oplus} ;$ S6: $50 \%$ resíduo $+25 \%$ CAC + 25\% Rendimax Floreira ${ }^{\oplus} ;$ S7: $75 \%$ resíduo $+12,5 \%$ CAC $+12,5 \%$ Rendimax Floreira ${ }^{\circledR}$. ${ }^{2}$ CRA: Capacidade Retenção de Água; ${ }^{3}$ DU: Densidade úmida; ${ }^{4}$ DS: Densidade seca. Fonte: Centro de Solos e Recursos Agroambientais. Laboratório de Análise de Solo e Planta, IAC - Campinas, SP, 2007. 
considerando-se os materiais isoladamente, observa-se que o Rendimax Floreira ${ }^{\circledR}$ foi aquele que mais se aproximou da faixa ideal para a maioria das culturas, por ser substrato comercialmente vendido para pronto uso, apresentando condutividade elétrica de $1,90 \mathrm{dS} \mathrm{m}^{-1}$, enquanto o resíduo (S1) e a CAC apresentaram valores baixos de condutividade elétrica, segundo Ballester-Olmos (1993).

Quanto aos nutrientes minerais, os substratos S2, S4 e S5 apresentaram maiores teores de nitrato $\left(\mathrm{NO}_{3}{ }^{-}\right)$, enxofre $(\mathrm{S})$, cálcio $(\mathrm{Ca})$ e magnésio $(\mathrm{Mg})$, uma vez que na sua composição foram adicionadas maiores proporções do Rendimax Floreira ${ }^{\circledR}$, o que contribuiu, em parte, no fornecimento destes nutrientes. Quando analisados os materiais individualmente, a CAC apresentou maiores teores de fósforo $(\mathrm{P})$ e potássio (K), o que também foi observado por Guerrini \& Trigueiro (2004), enquanto o resíduo de mineração de areia foi o que apresentou menores teores dos nutrientes, exceto para o nitrogênio amoniacal $\left(\mathrm{NH}_{4}^{+}\right)$.

Para as características físicas, quando os materiais foram analisados individualmente, a CAC e o Rendimax Floreira ${ }^{\circledR}$ apresentaram alta capacidade de retenção de água (Tabela 2); porém, quando utilizados juntos nos substratos (S2, S4, S5, S6 e S7) promoveram redução da capacidade de retenção de água, favorecendo a drenagem do excesso hídrico e o aumento da aeração do substrato e, consequentemente, maior desenvolvimento em altura e diâmetro das mudas de bananeira (Tabela 3). Klein et al. (2002), quando avaliaram as alterações nas propriedades físico-hídricas de substratos comerciais, com a mistura de CAC em diferentes proporções, concluíram que este material pode ser utilizado para otimizar as propriedades físico-hídricas de substratos hortícolas, melhorando a disponibilidade de água às plantas e a porosidade de aeração. O resíduo de mineração de areia (S1) apresentou baixa capacidade de retenção de água, por apresentar em sua composição maiores quantidades de grânulos de areia. Poole \& Waters (1972) e Paul \& Lee (1976) tentaram obter porosidade ideal para a planta, possibilitando o fornecimento de água e oxigênio de maneira adequada, pelo ajuste da taxa de constituintes de substratos.

Considerando-se que no presente trabalho foram utilizados sacos plásticos com cerca de $15 \mathrm{~cm}$ de altura, somente os substratos S2, S6 e o Rendimax Floreira ${ }^{\circledR}$ ficaram dentro da faixa considerada ideal para densidade seca e, os substratos S3, S4, S5 e S7, muito próximos da faixa considerada ideal por Kämpf (2000b), que apresentaram valores de 250 a $400 \mathrm{~kg} \mathrm{~m}^{-3}$ para recipientes de até $15 \mathrm{~cm}$ de altura, de 300 a $500 \mathrm{~kg} \mathrm{~m}^{-3}$ para recipientes de 20 a $30 \mathrm{~cm}$ de altura e de 500 a $800 \mathrm{~kg} \mathrm{~m}^{-3}$ para recipientes maiores. Para a escolha do substrato a ser utilizado em recipientes, a principal propriedade física a ser considerada é a densidade, sendo que, quanto menor o recipiente, mais baixa deve ser a sua densidade (Fermino, 2002).
Foram observadas diferenças para as variáveis de desenvolvimento avaliadas em mudas de bananeira 'Grande Naine'. Para a altura e diâmetro do colo das plantas, os substratos S2, S4, S5, S6 e S7 foram os que apresentaram resultados estatisticamente superiores, em comparação com os demais. Estes tratamentos (S2, S4, S5, S6 e S7) possuíam em sua composição o substrato comercial Rendimax Floreira ${ }^{\circledR}$, que apresentou resultados superiores aos dos demais para a maioria dos nutrientes analisados, fato que pode ter influenciado positivamente o desenvolvimento das mudas de bananeira. Por outro lado, os substratos $\mathrm{S} 1$ e S3 foram os que apresentaram resultados inferiores, com diferenças para menos, quando comparados com os dos outros substratos, o que pode estar relacionado com a presença de resíduo e resíduo + CAC, em suas respectivas composições que apresentaram menores teores de nutrientes.

Os substratos S1, S2, S4, S5, S6 e S7 foram os que resultaram em plantas com maior número de folhas e maior massa de matéria seca da parte aérea, sem apresentar diferenças entre si, porém superiores estatisticamente aos do substrato S3 (Tabela 3), pois neste substrato observaramse, nos resultados da análise química, baixos teores de $\mathrm{NH}_{4}^{+}$, $\mathrm{S}, \mathrm{Ca}, \mathrm{Mg}$ e boro (B), em vista da baixa contribuição dos materiais que o compõe (resíduo e CAC). De acordo com Moreira et al. (2006), o número total de folhas na planta é muito importante, pois, frequentemente, mudas com maior número têm maior índice de pegamento no campo, pois são as estruturas responsáveis pela captação de energia solar e produção de matéria orgânica por fotossíntese.

Para massa de matéria seca das raízes, houve menor acúmulo no substrato S3, sem apresentar, porém, diferenças, comparativamente às mudas produzidas com os substratos S1, S4, S6 e S7 (Tabela 3). O substrato S1 (resíduo de areia) apresentou baixos teores de nutrientes $\left(\mathrm{NO}_{3}^{-}, \mathrm{K}\right.$ e $\left.\mathrm{Mg}\right)$ e, quando misturado com a CAC (S3), apresentou aumentos nos teores destes nutrientes, porém, tais aumentos não supriram as necessidades nutricionais para as mudas de bananeira, prejudicando o desenvolvimento radicular. Os substratos S6 e S7 apresentam em sua composição o substrato comercial Rendimax Floreira ${ }^{\circledR}$ e a CAC em quantidades inferiores a $25,0 \%$, o que prejudicou o desenvolvimento radicular das mudas de bananeiras, visto que estes materiais apresentaram altos teores de nutrientes, principalmente $\mathrm{NO}_{3}^{-}, \mathrm{P}$, $\mathrm{K}, \mathrm{Ca}, \mathrm{Mg}$ e $\mathrm{S}$.

A partir destes resultados, observa-se a importância do acréscimo de material orgânico (Rendimax Floreira ${ }^{\circledR}$ ) na formulação de substratos, pois ele favorece a obtenção de características físicas e químicas adequadas para o desenvolvimento das plantas. Estes resultados corroboram os obtidos por Moreira et al. (2006), que estudaram o efeito de diferentes substratos formulados a partir de 
Tabela 3. Altura da planta (AP), diâmetro do colo (DC), número de folhas (NF), massa da matéria seca da parte aérea (MMSPA) e das raízes (MMSR) de mudas de bananeira 'Grande Naine' em função dos diferentes substratos, aos 98 dias de aclimatização

\begin{tabular}{lccccc}
\hline Substratos $^{1}$ & AP $(\mathbf{c m})$ & DC $(\mathbf{c m})$ & NF & MMSPA (g) & MMSR (g) \\
\hline S5 & $23,4 \mathrm{a}$ & $2,8 \mathrm{a}$ & $8,0 \mathrm{a}$ & $20,7 \mathrm{a}$ & $5,3 \mathrm{a}$ \\
S6 & $23,3 \mathrm{a}$ & $2,7 \mathrm{a}$ & $8,0 \mathrm{a}$ & $18,9 \mathrm{ab}$ & $4,3 \mathrm{abc}$ \\
S4 & $23,0 \mathrm{a}$ & $2,6 \mathrm{a}$ & $7,5 \mathrm{ab}$ & $16,5 \mathrm{ab}$ & $4,6 \mathrm{abc}$ \\
S2 & $22,9 \mathrm{a}$ & $2,7 \mathrm{a}$ & $8,0 \mathrm{a}$ & $20,7 \mathrm{a}$ & $5,1 \mathrm{ab}$ \\
S7 & $22,1 \mathrm{a}$ & $2,6 \mathrm{a}$ & $7,3 \mathrm{ab}$ & $21,2 \mathrm{a}$ & $4,5 \mathrm{abc}$ \\
S3 & $20,1 \mathrm{~b}$ & $2,4 \mathrm{~b}$ & $7,0 \mathrm{~b}$ & $14,2 \mathrm{~b}$ & $3,1 \mathrm{c}$ \\
S1 & $19,7 \mathrm{~b}$ & $2,4 \mathrm{~b}$ & $7,3 \mathrm{ab}$ & $18,0 \mathrm{ab}$ & $3,5 \mathrm{bc}$ \\
\hline CV $(\%)$ & 3,2 & 3,1 & 4,9 & 14,2 & 18,0 \\
\hline
\end{tabular}

${ }^{1}$ S1: $100 \%$ resíduo; S2: 50\% resíduo + 50\% Rendimax Floreira ${ }^{\oplus}$; S3: 50\% resíduo + 50\% CAC; S4: 50\% CAC + 50\% Rendimax Floreira ${ }^{\oplus ;}$ S5: 25\% resíduo + 37,5\% CAC + 37,5\% Rendimax Floreira ${ }^{\oplus} ;$ S6: 50\% resíduo + 25\% CAC + 25\% Rendimax Floreira ${ }^{\circledR} ;$ S7: 75\% resíduo + 12,5\% CAC $+12,5 \%$ Rendimax Floreira ${ }^{\circledR}$. Médias seguidas da mesma letra não diferem entre si, a 5\% de probabilidade pelo teste Tukey.

solo, esterco bovino, Plantmax e composto orgânico, para a aclimatização de mudas micropropagadas de abacaxizeiro 'Pérola', cujos resultados que evidenciaram a necessidade de se ter proporções adequadas de componentes orgânicos nos substratos, para permitir adequado desenvolvimento das plantas, visto que o substrato exerce influência significativa na arquitetura do sistema radicular, no estado nutricional das plantas e no movimento de água no sistema solo-planta-atmosfera.

\section{CONCLUSÃO}

Concluiu-se que o resíduo de mineração de areia pode ser recomendado para a aclimatização de mudas de bananeira 'Grande Naine', desde que usado em mistura com outros materiais ou substratos, com limite máximo de $75 \%$ de inclusão.

\section{REFERÊNCIAS}

Ballester-Olmos JF (1993) Substratos para el cultivo de plantas ornamentales. Madrid, Saijen. 44p.

Borges AL, Alves EJ, Silva SO \& Souza LS (1997) O cultivo da banana. Cruz das Almas, EMBRAPA-CMPMF. 109p. (Circular Técnica, 27).

FAOSTAT (2010) Agricultural data. Disponível em: <http:// faostat.fao.org> Acessado em: 24 de maio 2010.

Fermino MH (2002) O uso da análise física na avaliação da qualidade de componentes e substratos. In: Encontro Nacional de Substratos para Plantas, Campinas. Anais, IAC-Campinas, p.29-37.

Ferreira DF (2003) SISVAR - Sistema para análise de variância. Lavras, Universidade Federal de Lavras - Departamento de Ciências Exatas (DEX), versão 5.5.

Gauland DCSP (1997) Relações hídricas em substratos à base de turfas sob o uso dos condicionadores casca de arroz carbonizada ou queimada. Dissertação de Mestrado. Universidade Federal do Rio Grande do Sul, Porto Alegre, 107p.

IBGE - Instituto Brasileiro de Geografia e Estatística. Disponível em: 〈http://www.sidra.ibge.gov.br〉. Acessado em: 12 janeiro 2010.

Kämpf AN (2000a) Seleção de materiais para uso como substratos. In: Kämpf AN \& Fermino MH (Eds.) Substratos para plantas: a base da produção vegetal em recipientes. Porto Alegre, Gênesis. p.139-145.
Guerrini IA \& Trigueiro RM (2004) Atributos físicos e químicos de substratos compostos por biossólidos e casca de arroz carbonizada. Revista Brasileira de Ciência do Solo, 28:1069-1076.

Kämpf NA (2000b) Produção comercial de plantas ornamentais. Guaíba, Agropecuária. 254p.

Klein VA, Camara RK, Simon MA \& Dias ST (2002) Casca de arroz carbonizada como condicionador de substrato. In: Furlani AMC (Ed.) Caracterização, manejo e qualidade de substratos para produção de plantas. Campinas, Instituto Agronômico. 95p. (Documentos IAC 70).

Lemos EEP, Ferreira MS, Alencar LMC, Oliveira JGL \& Magalhães VS (2001) Micropropagação de clones de banana cv. Terra em biorreator de imersão temporária. Revista Brasileira de Fruticultura, 23:482-487.

LUPA (2008) Levantamento Censitário das Unidades de Produção Agropecuária do Estado de São Paulo/ Secretaria de Agricultura e Abastecimento, CATI/IEA. Disponível em: <http:// www.cati.sp.gov.br/projetolupa> Acessado em: 10 de dezembro 2009.

Moreira MA, Carvalho JG, Pasqual M, Fráguas C \& Silva AB (2006) Efeito de substratos na aclimatação de mudas micropropagadas de abacaxizeiro cv. Pérola. Ciência e Agrotecnologia, 30:875879.

Moreira RS (1999) Banana: teoria e prática de cultivo. 2.ed. São Paulo, Fundação Cargill, 1999. CD ROM

Murashige T \& Skoog F (1962) A revised medium for rapid growth and bioassays with tobacco tissue cultures. Physiology Plantarum, 15:473-479.

Paul JL \& Lee CI (1976) Relation between growth of Chrysantemum and aeration of conterainer media. Journal of the American Society for Horticultural Science, 101:500-503.

Poole RT \& Waters WE (1972) Evaluation of various pottings media for growth of foliage plants. Proceedings of the Florida State Horticultural Society, 85:395-398.

Schmitz JAK, Souza PVD \& Kämpf AN (2002) Propriedades químicas e físicas de substratos de origem mineral e orgânica para o cultivo de mudas em recipientes. Ciência Rural, 32:937-944.

Sousa HU, Silva CRR, Carvalho JG \& Menegucci JLP (2000) Nutrição de mudas de bananeira em função de substratos e doses de superfosfato simples. Ciência e Agrotecnologia, 24:64-73.

Yamanishi OK, Fagundes GR, Machado Filho JA \& Valone GV (2004) Efeito de diferentes substratos e duas formas de adubação na produção de mudas de mamoeiro. Revista Brasileira de Fruticultura, 26:276-279. 